\title{
DESPLAZAMIENTO DE LA CIUDAD DE ALICANTE HACIA EL OESTE EN LOS ÚLTIMOS AÑOS
}

\author{
$\mathrm{M}^{\mathrm{a}}$ José Santos Deltell
}

\section{RESUMEN}

El centro de la ciudad de Alicante ha variado de emplazamiento de un tiempo a esta parte. En la década de los años cincuenta la Explanada de España y la Rambla eran los ejes horizontal y vertical respectivamente que vertebraban la centralidad del plano de Alicante.

La expansión demográfica de los años sesenta supuso una búsqueda de nuevos espacios destinados a albergar una población joven. De esta manera se fue formando un nuevo barrio denominado «Ensanche» en los alrededores de la antigua carretera de Alicante a Madrid convertida así en la avenida de Maisonnave.

Esta habitado fundamentalmente por familias jóvenes. La edad de los cabezas de familia oscila entre los treinta y los cuarenta años. Se trata de un colectivo cuyas actividades profesionales suelen ser liberales, tienen un poder adquisitivo medio-alto debido a la percepción de ingresos por parte de ambos cónyuges, en el seno de una misma familia.

Por el contrario, el centro histórico sufre una pérdida acusada de población y un envejecimiento progresivo de la misma, lo que se traduce en un abandono cada vez más frecuente de viviendas habitadas.

\section{RESUMEE}

Le centre tradicionel de la ville d'Alicante a change de lieu dans les dernieres aneés. On voit qu'il s'etait deplacé vers l'occident de la capital, oú s'était formée la banlieue nouvelle de l'Ensanche.

Dans cet endroit est oú habitent des jeunes familles avec deux fils genéralment. L'âge moyenne des parents se trouve autour des quarante ans. Ils ont des profesions liberals, la plus part avec un niveau adquisitive notable. 
Par contre, la vieille-ville suffre une perte de populatión prógresive á cause de leur enveillement, ce qui est une caracteristique á remarquer.

Se trata de poner de manifiesto la movilidad social que ha experimentado la población de la ciudad de Alicante, en los últimos años.

Es harto conocido que el centro tradicional de la ciudad se ha desplazado hacia el oeste. Lo que llamamos Casco Antiguo quedaba configurado por los barrios de Santa Cruz, Ayuntamiento, Raval Roig, Virgen del Socorro, Centro, Mercado y San Antón cuyos ejes viarios más importantes desde principios de siglo eran con dirección N-S la Rambla de Méndez Núñez y de E-W la Explanada de España que se cortan perpendicularmente.

La extensión del plano urbano de Alicante se ha ido prolongando a través de lo que se conoce con el nombre de «Ensanche», cuyos proyectos iniciales datan ya de fines del pasado siglo. Se trata del barrio constituido por una serie de calles homogéneas y rectilíneas, definidas y tiradas a cordel, que forman un plano en cuadrícula al estilo de los barrios de Salamanca, en Madrid y Cerdá en Barcelona.

Los límites de este ensanche serían: por el noroeste la estación del ferrocarril Alicante-Madrid, por el norte la calle de Benito Pérez Galdós hasta el Paseo de Marvá. Por el este una línea recta de norte a sur formada por una serie de paseos: el anteriormente citado, la plaza de los Luceros, Soto, Gadea y Canalejas, éste último perpendicular a los anteriores desemboca en el mar por el puerto pesquero. El sur lo delimita el Paseo de Canalejas y la Avenida de Loring. El cuadrilátero queda cerrado al oeste por otro gran eje viario trazado de norte-sur, la calle de Oscar Esplà.

El centro neurálgico de este gran barrio de Alicante se ha configurado alrededor de la instalación de unos grandes almacenes comerciales, El Corte Inglés en la intersección de las calles de Oscar Esplà y Maisonnave ${ }^{1}$. Esta última atraviesa en diagonal el Ensanche de este-oeste, enlazando los paseos de Soto y Gadea con la antigua carretera de Madrid.

Desde la apertura de este centro en 1989 toda la zona que le rodea ha cobrado nueva vida. Así han proliferado los servicios de bares, restaurantes, cafeterías, bancos, aparcamientos y tiendas en sus contornos. Nuevos edificios de viviendas han sido construidos en los últimos años, muchos de ellos de lujo, siendo un reclamo comercial y especulativo el que determinadas edificaciones se encontrarán en las proximidades de este gran comercio.

Con anterioridad a la ubicación de esta cadena comercial en la zona, este sector de la ciudad y sus aledaños eran simplemente zonas de almacenaje para los productos que se comerciaban por el puerto de Alicante, principalmente salazones,

1 El Corte Inglés fue inaugurado en Alicante en octubre de 1989. 
vinos y maderas. Debido a la buena comunicación existente entre la zona portuaria del muelle de Levante y la estación de ferrocarril Alicante-Madrid.

La planificación y posterior realización del Ensanche en Alicante se hizo pensando que sería el lugar idóneo donde se ubicaría la burguesía local, como había ocurrido en otras ciudades, sin embargo en el caso de Alicante no ocurrió así. Las familias más acomodadas no eligieron esta zona residencial para trasladarse a vivir sino que optaron por establecer sus viviendas en el frente costero.

En Alicante a finales del XIX y según los planos del arquitecto Guardiola Picó se había trazado el precioso Paseo de la Explanada frente al mar $(1868)^{2}$. Se extendía desde la puerta del Mar hasta el Parque de Canalejas, antiguo varadero. Todo él estaba plantado de palmeras, lo que le daba un aspecto muy exótico.

Así de esta manera se fueron construyendo edificios que hoy en día forman parte de la personalidad e idiosincrasia de la ciudad: Casa Carbonell, Lamagniere, Alberola, ésta última mutilada en parte y adosada a un edificio de trazado moderno, que rompe todas las proporciones estéticas imaginables.

\section{El barrio del ensanche}

El panorama urbano de Alicante en solo seis años ha variado de manera radical. El alto valor alcanzado por el suelo desde la instalación de El Corte Inglés y la especulación de que ha sido objeto en los alrededores de estos grandes almacenes ha contribuido a generar un aumento del número de comercios con diferente especialización según sectores, así por ejemplo la calle de Pintor Aparicio se ha dedicado en su mayor parte a la confección de ropa al por mayor.

Los altos valores alcanzados por el metro cuadrado de edificación, en locales comerciales han superado a veces los tres millones de pesetas, y las 300.000 pesetas metro cuadrado en las viviendas. Ello ha contribuido a mejorar el aspecto urbanístico de la ciudad.

El bulevar de Oscar Esplà con un paseo central rodeado de plantas y árboles ofrece a ambos lados una sucesión de edificios nuevos y modernos de altas calidades cuyos bajos suelen estar ocupados mayoritariamente por bancos, cajas de ahorros y entidades de seguros.

Este paseo abierto por donde discurría el barranco de Benalua, se ha trazado de norte a sur desde la plaza abierta en la intersección de las calles (Avenida de Aguilera, Avenida de Salamanca, Avenida de Maisonnave y la anteriormente citada) hasta la estación de Murcia y el mar. Configura el límite occidental de este barrio.

2 «la realización de esta obra debe inscribirse en la ideología higienista de la época que pretendía la conformación de un paseo marítimo a lo largo de la totalidad de la fachada costera de la ciudad, abarcando desde el Parque de Canalejas hasta el Paseo del Postiguet...» (RAMOS HIDALGO, A. 1984). 
Atraviesa el paseo de Oscar Esplà, la Avenida de Eusebio Sempere y la de Catedrático Soler, con dirección noroeste-sureste. El sur del cuadrilátero lo forman los accesos al puerto, Avenida de Loring desde la estación de Murcia, Plaza de Galicia, Puerto pesquero, Depósito franco, Parque de Canalejas, y Avenida de Ramón y Cajal.

Los edificios que presentan fachada al mar constituyen el último tramo del paseo «higienista» construido a finales del pasado siglo, la Explanada de España.

Por el este se suceden de norte a sur una serie de paseos de trazado cuidado y armonioso que culminan en la escalinata del I.N.B. Jorge Juan (Paseo de Marva, Plaza de los Luceros. Soto, y Gadea). Los edificios alternan con comercios de los más representativos de la ciudad.

El sector norte queda limitado por la Avenida de la Estación denominada así porque se abre desde la estación de Renfe hasta la plaza de los Luceros. En ella se encuentra el Palacio de la Diputación Provincial.

La Avenida de Maisonnave atraviesa el plano del Ensanche de noroeste a sureste prácticamente a la mitad de la cuadrícula. Esta amplia arteria del callejero moderno, constituía en otro tiempo el acceso a la carretera Alicante-Madrid y estaba flanqueada a ambos lados por almacenes de maderas y solares. Desde los años ochenta el desarrollo espectacular de esta gran vía ha alcanzado cotas elevadísimas. Hoy en día su aspecto ha variado notablemente, de aquella destartalada carretera se ha pasado a una amplia calle rodeada de edificios modernos y un gran número de comercios. Destacan aquí las consultas médicas de los especialistas más afamados de la ciudad. El subterráneo de toda la avenida esta dedicado a albergar un aparcamiento de automóviles en un gran parking.

La densidad de tráfico y viandantes en toda la zona es desorbitada, sobre todo por las tardes y en horario comercial; este aspecto multitudinario y físicamente agradable y bonito contrasta con el de la ciudad antigua que parece adormecida y deshabitada.

El casco antiguo de Alicante es la reserva de núcleos de inmigrantes marroquíes, que en su mayor parte buscan alojamientos baratos y una mayor proximidad al puerto. Como conclusión se puede establecer una gran diferencia entre ambos núcleos urbanos, el antiguo y el moderno, el oriental y el occidental. El antiguo originado en las estribaciones del Benacantil tiene carácter residual y obsoleto, es testigo mudo de otros tiempos y forma parte de la historia de la ciudad.

Por el contrario, el nuevo abierto y trazado sobre el llano ha conseguido imprimir el sello de la modernidad a Alicante, siendo esta una de las poblaciones mas atractivas de España en la actualidad.

El Ensanche no había cumplido totalmente la función a la que había sido destinada, la de albergar un contingente de población numeroso, hasta la construcción del establecimiento citado.

Su creación entre las calles de Maisonnave, Pintor Aparicio, Castellar y Chu- 


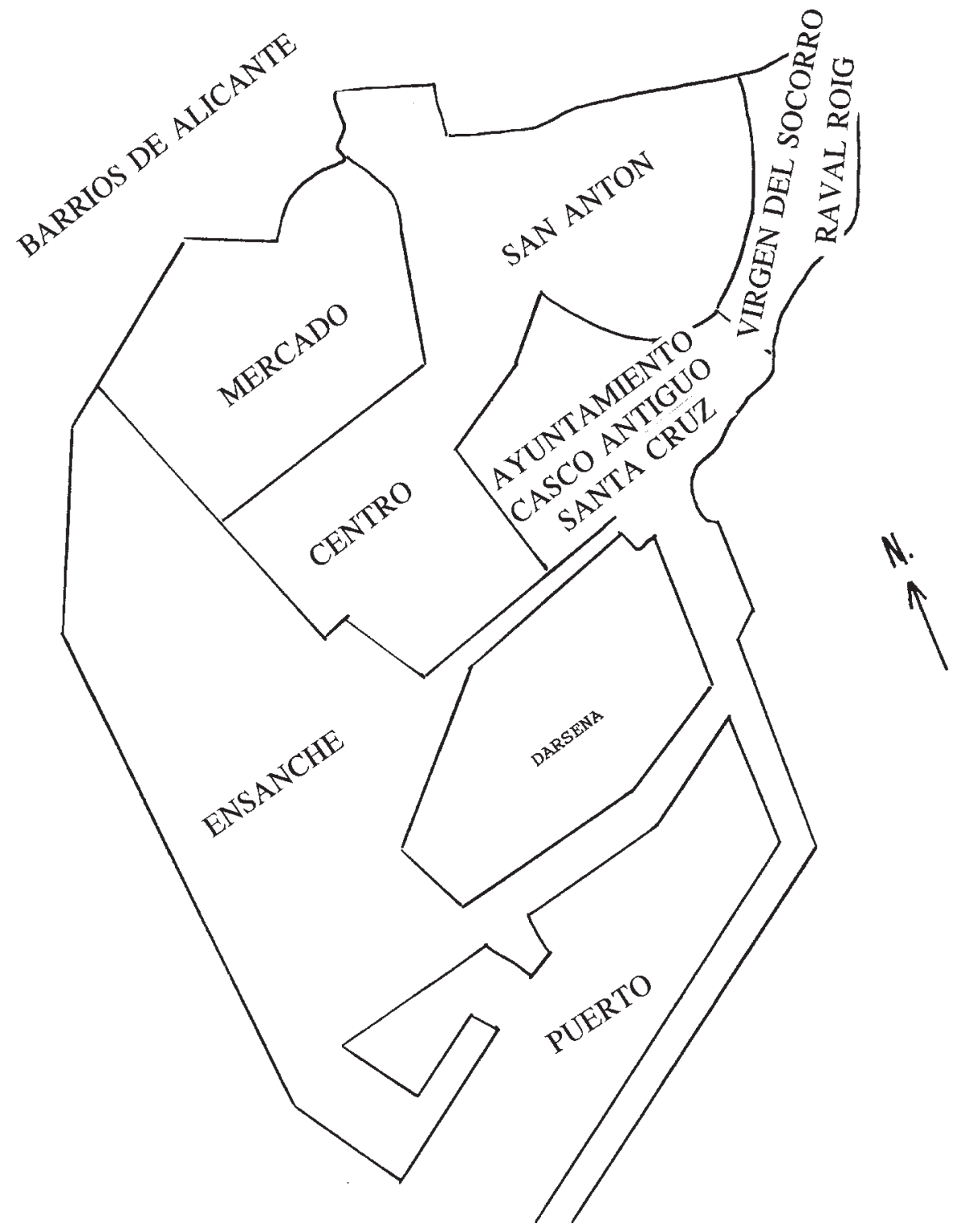

rruca (norte, este, sur y oeste respectivamente), ha sido un factor decisivo que ha favorecido la expansión de edificaciones por el Ensanche. Es de destacar que la población que habita en las zonas limítrofes forma parte de la clase media alicantina, suelen ser familias jóvenes, constituidas generalmente por cuatro miembros (padres y dos hijos), en la mayor parte de los casos ambos cónyuges trabajan, son universitarios y cuentan con un alto poder adquisitivo lo que favorece la función comercial de la zona (gráficos 1 y 2). 


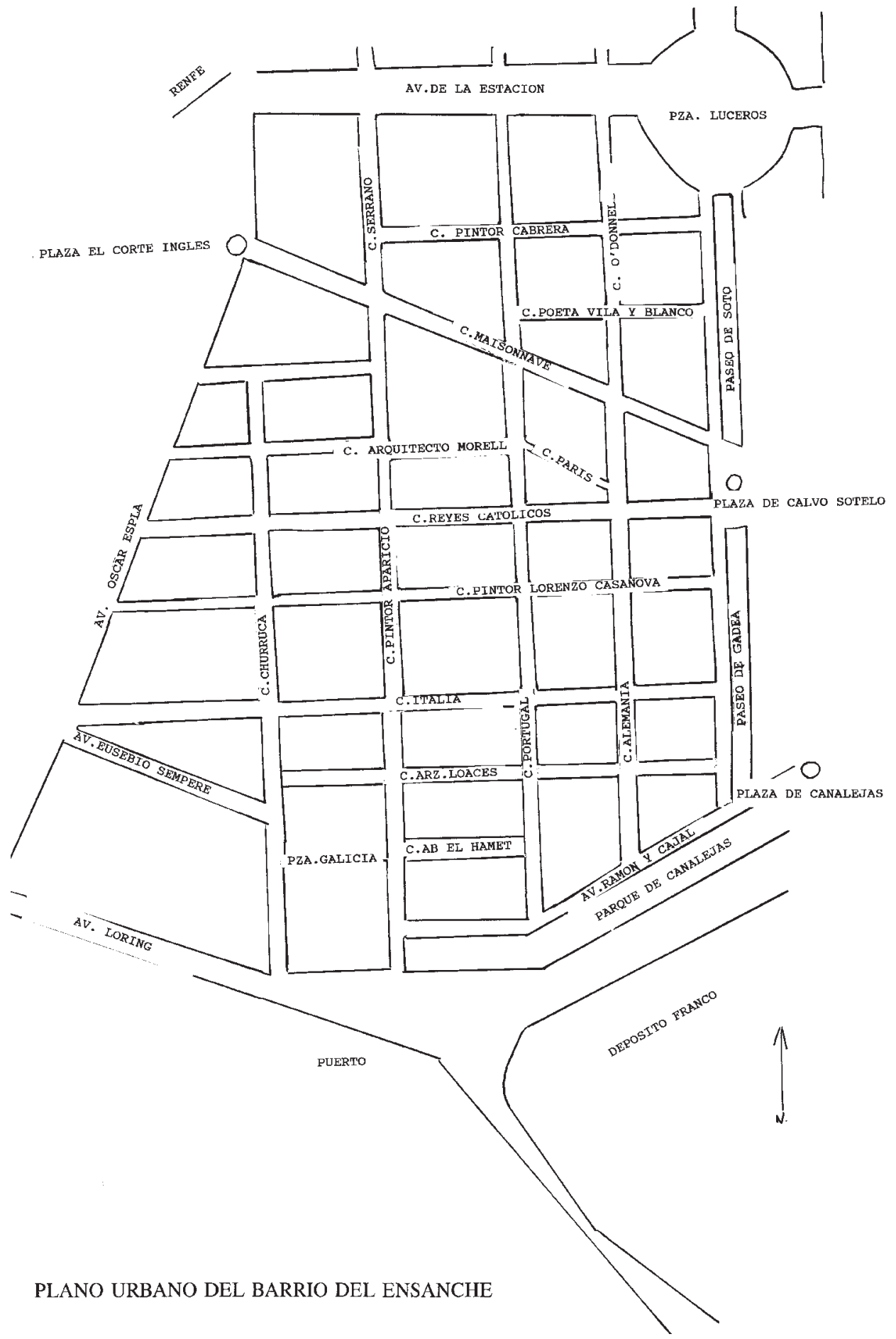


Cuadro I. EVOLUCIÓN DE LA POBLACIÓN

\begin{tabular}{|lcccc|}
\hline & $\mathbf{1 9 8 6}$ & $\mathbf{1 9 9 1}$ & Diferencia & Crecimiento \\
\hline Casco antiguo $^{3}$ & 25.425 & 23.784 & 1641 & $-7 \%$ \\
Ensanche $^{4}$ & 18.439 & 19.400 & 961 & $5 \%$ \\
\hline
\end{tabular}

Como se desprende del cuadro I la evolución de la población en la ciudad de Alicante se ha ido desplazando espacialmente en los últimos años, siendo el crecimiento negativo para los barrios del Casco Antiguo (-7\%) y positivo para el Ensanche (5\%) entre 1986-91.

\section{Cuadro II. DISTRIBUCIÓN POR SEXOS}

\begin{tabular}{|lcccccc|}
\hline & \multicolumn{2}{c}{$\mathbf{1 9 8 6}$} & \multicolumn{4}{c|}{1991} \\
& hombres & mujeres & Totales & hombres & mujeres & Totales \\
\hline Casco Antiguo & 11.460 & 13.965 & 25.425 & 10.847 & 12.937 & 23.784 \\
Ensanche & 8.649 & 9.790 & 18.439 & 9.100 & 10.300 & 19.400 \\
\hline
\end{tabular}

En cuanto a la diferenciación por sexos en el casco antiguo tradicional predomina la población femenina (mayor sobremortalidad masculina) mientras que en el ensanche por ser un barrio de gente más joven ambos sexos se encuentran más igualados.

Cuadro III. LUGAR DE NACIMIENTO

\begin{tabular}{|lrrrr|}
\hline & Casco Antiguo & \multicolumn{1}{c}{$\%$} & Ensanche & \multicolumn{1}{c|}{$\%$} \\
\hline Alicante & 12.040 & $50,6 \%$ & 8.402 & $43,3 \%$ \\
Provincia & 3.240 & $13,6 \%$ & 2.744 & $14,1 \%$ \\
C.Valenciana & 638 & $2,6 \%$ & 754 & $3,8 \%$ \\
España & 6.684 & $28,3 \%$ & 6.704 & $34,8 \%$ \\
Extranjero & 1.182 & $4,9 \%$ & 796 & $4,0 \%$ \\
\hline TOTALES & 23.784 & 100,00 & 19.400 & $100,00^{5}$ \\
\hline
\end{tabular}

Fuente: E.P. Padrón 1991, Alicante.

3 Comprende lo que denominamos «Casco Antiguo» los barrios de: Santa Cruz, Ayuntamiento, Raval Roig, Virgen del Socorro, Centro, Mercado y San Antón.

4 El llamado Ensanche corresponde a la expansión decimonónica de Alicante: DiputaciónRenfe, Séneca-Autobuses y Plaza de Galicia.

5 Datos del patrón de habitantes de 1991. 
Según el lugar de nacimiento, la mayor proporción de nacidos corresponde a la ciudad de Alicante para ambos espacios. La cifra se incrementa ligeramente en el Casco Antiguo 50,6\% frente a un 43,3\% en el Ensanche. Sin embargo la población inmigrante o proveniente de otras ciudades españolas es mayor en el Ensanche que en el Casco Antiguo 34,8\% frente a un 28,3\% como corresponde a un barrio de reciente creación. Los demás porcentajes están bastante igualados, para los que han nacido en el resto de la provincia, Comunidad Valenciana o los que desbordan las fronteras nacionales.

\section{Cuadro IV. TITULACIÓN}

\begin{tabular}{|lrrrrr|}
\hline \multicolumn{2}{r}{ Casco Antiguo } & $\%$ & Ensanche & $\%$ & TOTALES \\
\hline No saben leer & 211 & 80,5 & 51 & 19,5 & 262 \\
Sin estudios & 3,711 & 68,6 & 1693 & 31,4 & 5.404 \\
EGB & 3.943 & 53,8 & 3381 & 46,2 & 7.324 \\
BUP & 3.581 & 48,9 & 3729 & 51,1 & 7.310 \\
Licenciados & 1.612 & 44,5 & 2006 & 55,5 & 3.618 \\
\hline
\end{tabular}

Respecto al nivel de instrucción y titulación las diferencias son quizás las más significativas. Mientras que en el grupo de barrios del Casco Antiguo un 80,55\% no saben leer ni escribir, y un 68,6\% no tienen estudios, en el Ensanche la proporción de gente con estudios es muy superior alcanzando niveles que traspasan ampliamente el umbral del $50 \%$ en los que cuentan con el BUP 51,1\% y Licenciados 55,5\%.

Cuadro V. EDAD QUE PREDOMINA EN LOS CABEZAS DE FAMILIA

\begin{tabular}{|lrrrr|}
\hline & Edad & Hogares* & Total** & $\%$ \\
\hline Casco Antiguo & & & & \\
1 & $65-69$ & 138 & 1173 & 11,7 \\
2 & $60-64$ & 71 & 604 & 11,7 \\
3 & $65-69$ & 333 & 2480 & 13,4 \\
4 & $65-69$ & 408 & 3602 & 11,3 \\
5 & $65-69$ & 120 & 959 & 12,5 \\
Ensanche & & & & 22,7 \\
& $40-49$ & 1423 & 6247 & \\
\hline
\end{tabular}

1. Barrios de Santa Cruz y Ayuntamiento; 2. Virgen del Socorro y Raval Roig; 3. Centro; 4. Mercado; 5. San Antón.

* Hogares cuyo cabeza de familia tiene esa edad.

** Total de hogares por barrio. 
Cuadro VI

NÚMERO DE MIEMBROS POR FAMILIA

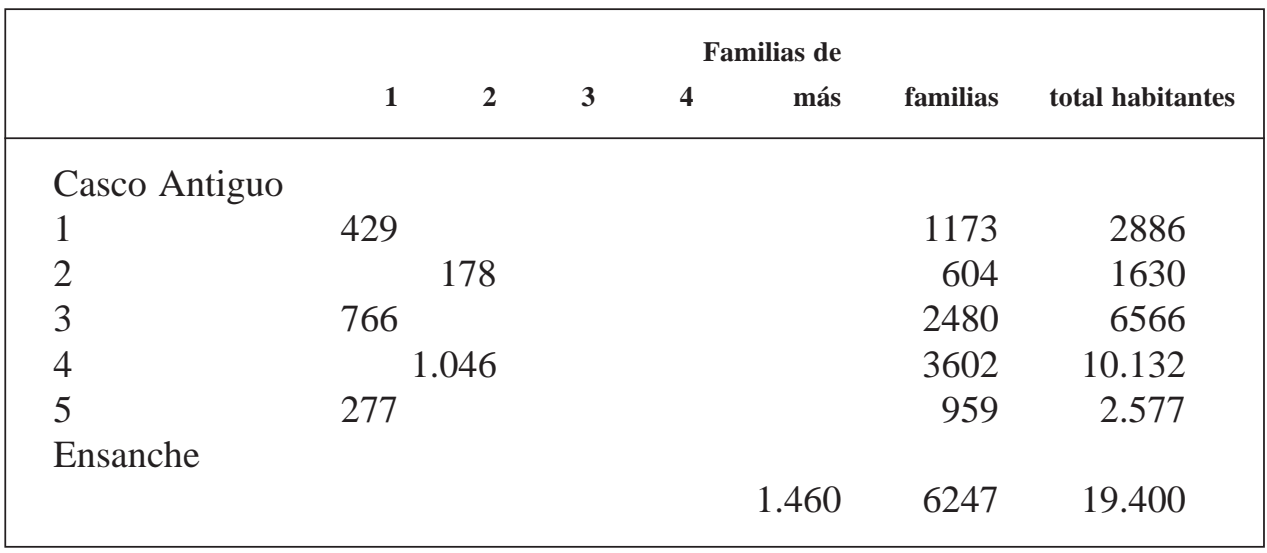

De igual manera las desigualdades son notorias en cuanto a la edad que ostenta el cabeza de familia. En los barrios antiguos la edad media oscila entre los 60-69 años en todos los casos con porcentajes superiores al $10 \%$, lo que determina una población envejecida. En su mayor parte se trata de jubilados o pensionistas con niveles de renta muy bajos. En el Ensanche la edad de la persona principal se reduce en veinte años, siendo la media de 40-49 años con un 22,7\% lo que supone que casi una cuarta parte de la población queda comprendida entre esos umbrales dando lugar a una población joven en su mayor parte.

\section{Cuadro VII}

NÚMERO DE ESTABLECIMIENTOS DE LAS CALLES LIMÍTROFES A EL CORTE INGLÉS

\begin{tabular}{|lrrrrrrrrrrr|}
\hline & C/ Castellar & C/ Churruca & C/ Maisonnave & C/Oscar Espla & C/ Pintor Aparicio \\
\cline { 2 - 10 } Establecimiento & 1987 & $\mathbf{1 9 9 4}$ & $\mathbf{1 9 8 7}$ & $\mathbf{1 9 9 4}$ & $\mathbf{1 9 8 7}$ & $\mathbf{1 9 9 4}$ & $\mathbf{1 9 8 7}$ & $\mathbf{1 9 9 4}$ & $\mathbf{1 9 8 7}$ & $\mathbf{1 9 9 4}$ \\
\hline Adm. y Servicios & - & - & 3 & 4 & 4 & 19 & 1 & 14 & 2 & 6 \\
Autoescuelas & 1 & 1 & - & - & - & 2 & - & - & - & 2 \\
Bancos & - & - & - & 1 & 2 & 2 & 1 & 5 & - & 1 \\
Comercios & 1 & 5 & 14 & 27 & 2 & 19 & 8 & 22 & 18 & 37 \\
Consultas médicas & - & 1 & - & 6 & 1 & 10 & - & 2 & 1 & 2 \\
Espectáculos & - & - & - & - & - & - & - & - & 1 & 1 \\
Hostelería y Alimentación & - & 2 & 5 & 7 & 5 & 6 & 4 & 10 & 1 & 3 \\
Peluquería y estética & - & - & 1 & 2 & - & 5 & - & 1 & - & 2 \\
\hline Totales & 2 & 9 & 23 & 47 & 14 & 63 & 14 & 54 & 23 & 54 \\
\hline
\end{tabular}


Finalmente las disparidades se establecen también en cuanto al número de miembros por familia. En los barrios tradicionales la media queda comprendida entre una y dos personas por vivienda, lo que da origen a un despoblamiento paulatino por envejecimiento y defunción. Con el consiguiente deterioro de los barrios que se van quedando anticuados y sin vida.

Mientras que en el Ensanche, la media por unidad familiar se establece en cuatro miembros, que suelen ser los progenitores y dos hijos.

\section{Conclusiones}

En tan sólo cinco años de 1986 a 1991 se observan cambios significativos en cuanto a dinamismo en la zona del Ensanche, favorecidos sin duda por todos esos factores comerciales que han servido para potenciar la situación y crear un ambiente privilegiado para los grupos de gente que viven en sus alrededores ${ }^{6}$.

Por el contrario el resto del centro de Alicante se va quedando anticuado y despoblado. Se asiste a un envejecimiento de la población, atraso cultural y niveles de renta per capita más bajos. Todo ello es síntoma de la dicotomía espacial en la que estamos inmersos en Alicante. Se podría decir que se trata de dos ciudades distintas.

Los organismos pertinentes que se encargan de enmascarar o difuminar esas diferencias tan manifiestas, intentan promocionar el denominado «Casco Antiguo» con campañas de publicidad, que elogian el comercio del «corazón de Alicante», los mercados municipales, exposiciones, congresos, museos y turismo para la tercera edad. Pero a pesar de todos los esfuerzos, la verdad es que el Alicante del año 2000 se ha desplazado hacia el Oeste.

6 Se ha llegado a denominar al suelo urbano de la calle Maisonnave de Alicante, «la milla de oro» con valores superiores a 3.000.000 pesetas/metro cuadrado. Por su similitud con la de Marbella donde tienen su residencia todos los famosos de la Jet internacional. 